\title{
History of POIC Capabilities and Limitations to Conduct International Space Station Payload Operations
}

\author{
Steven Dyer*, Rebecca Grimaldi*, Tim Horvath**, Denise Morris*, Patricia Patterson**, and \\ Emily Willis* \\ *ISS Payload Operations Director, NASA/EO03, Marshall Space Flight Center, Huntsville, \\ Alabama 35812 \\ **ISS Payload Operations Manager, NASA/EO03, Marshall Space Flight Center, Huntsville, \\ Alabama 35812
}

Payload science operations on the International Space Station (ISS) have been conducted continuously twenty-four hours per day, 365 days a year beginning February, 2001 and continuing through present day. The Payload Operations Integration Center (POIC), located at the Marshall Space Flight Center in Huntsville, Alabama, has been a leader in integrating and managing NASA distributed payload operations. The ability to conduct science operations is a delicate balance of crew time, onboard vehicle resources, hardware up-mass to the vehicle, and ground based flight control team manpower. Over the span of the last ten years, the POIC flight control team size, function, and structure has been modified several times commensurate with the capabilities and limitations of the ISS program. As the ISS vehicle has been expanded and its systems changed throughout the assembly process, the resources available to conduct science and research have also changed. Likewise, as ISS program financial resources have demanded more efficiency from organizations across the program, utilization organizations have also had to adjust their functionality and structure to adapt accordingly. The POIC has responded to these often difficult challenges by adapting our team concept to maximize science research return within the utilization allocations and vehicle limitations that existed at the time. In some cases, the ISS and systems limitations became the limiting factor in conducting science. In other cases, the POIC structure and flight control team size were the limiting factors, so other constraints had to be put into place to assure successful science operations within the capabilities of the POIC. This paper will present the POIC flight control team organizational changes responding to significant events of the ISS and Shuttle programs. 


\title{
History of POIC Capabilities and Limitations to Conduct International Space Station Payload Operations
}

\author{
Rebecca Grimaldi ${ }^{1}$, Tim Horvath ${ }^{2}$, Denise Morris ${ }^{1}$, Emily Willis ${ }^{1}$, Lamar Stacy ${ }^{2}$, \\ Mike Shell ${ }^{1}$, Mark Faust ${ }^{1}$, Jason Norwood ${ }^{1}$ \\ National Aeronautics and Space Administration, Huntsville, Alabama, 35812
}

\begin{abstract}
Payload science operations on the International Space Station (ISS) have been conducted continuously twenty-four hours per day, 365 days a year beginning February, 2001 and continuing through present day. The Payload Operations Integration Center (POIC), located at the Marshall Space Flight Center in Huntsville, Alabama, has been a leader in integrating and managing NASA distributed payload operations. The ability to conduct science operations is a delicate balance of crew time, onboard vehicle resources, hardware up-mass to the vehicle, and ground based flight control team manpower. Over the span of the last ten years, the POIC flight control team size, function, and structure has been modified several times commensurate with the capabilities and limitations of the ISS program. As the ISS vehicle has been expanded and its systems changed throughout the assembly process, the resources available to conduct science and research have also changed. Likewise, as ISS program financial resources have demanded more efficiency from organizations across the program, utilization organizations have also had to adjust their functionality and structure to adapt accordingly. The POIC has responded to these often difficult challenges by adapting our team concept to maximize science research return within the utilization allocations and vehicle limitations that existed at the time. In some cases, the ISS and systems limitations became the limiting factor in conducting science. In other cases, the POIC structure and flight control team size were the limiting factors, so other constraints had to be put into place to assure successful science operations within the capabilities of the POIC. This paper will present the POIC flight control team organizational changes responding to significant events of the ISS and Shuttle programs.
\end{abstract}

\section{The Payload Operations Integration Center (POIC)}

Payload science operations on the International Space Station (ISS) have been conducted continuously twentyfour hours per day, 365 days a year beginning February, 2001 and continuing through present day. The Payload Operations Integration Center (POIC), located at the Marshall Space Flight Center (MSFC) in Huntsville, Alabama, is the control center tasked with integrating and operating National Aeronautics and Space Administration (NASA) research facilities and science experiments on behalf of NASA and the ISS Program.

The POIC is one of five control centers around the world that supports ISS operations on a continuous basis. Each of the five centers performs a unique function in support of the crew that inhabits and hardware that comprises the ISS. See Figure 1- ISS Control Centers. NASA's Mission Control Center - Houston (MCC-H) is the lead control center for overall crew safety and ISS vehicle operations, and ensures each of the other four control centers is coordinated and fulfilling its designated tasks. The MCC-H is led by the Houston Flight Director. Mission Control Center - Moscow (MCC-M) is responsible for the Russian Segment of the ISS, including cosmonauts and hardware, as well as any cargo or other space vehicles that dock to the Russian Segment. Examples of other

\footnotetext{
${ }^{1}$ Payload Operations Director, Mission Operations Lab, EO03.

${ }^{2}$ Payload Operations Manager, Mission Operations Lab, EO03. 
spacecraft for which MCC-M is responsible for, are the Soyuz capsules and Progress cargo vehicles. The MCC-M is led by the Shift Flight Director.

The Columbus Control Center (COL-CC) is responsible for the European Space Agency's (ESA) Columbus module, including its hardware and any ESA sponsored experiments conducted within that module. The COL-CC is led by the Columbus Flight Director. The Space Station Integration and Promotion Center (SSIPC) is responsible for the Japanese Aerospace Exploration Agency's (JAXA) Kibo module, including its hardware and any JAXA sponsored experiments conducted within that module. The SSIPC is led by the JAXA Flight Director. The POIC control center is responsible for all NASA sponsored experiments conducted anywhere within ISS, including externally mounted facilities. The NASA experiments for which POIC is responsible may be conducted in NASA's Destiny Laboratory, Nodes, Columbus, or Kibo. The POIC is led by the Payload Operations Director (POD).

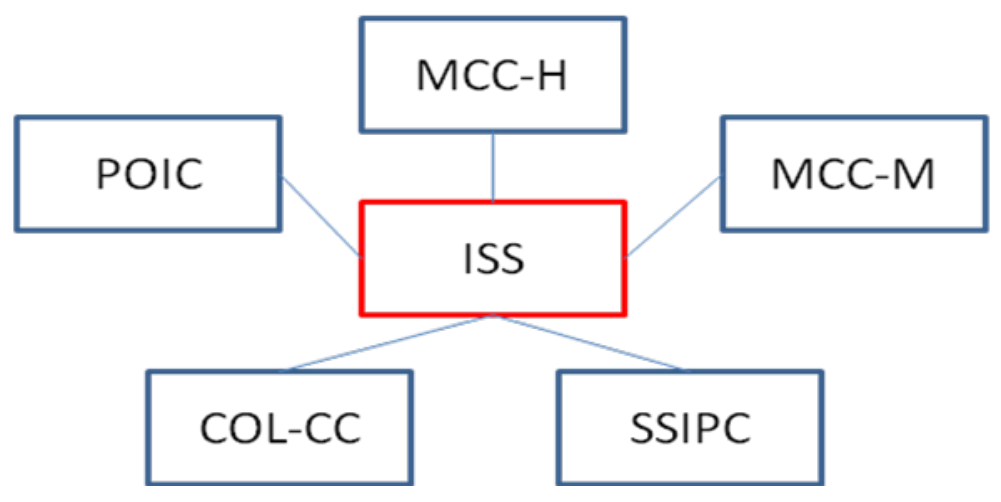

Figure 1 - ISS Control Centers

The support provided at POIC to the ISS, and to the ISS Program, consists of a real-time flight control team that staffs consoles around the clock, and a support team that works normal office hours. The functions performed by the real-time flight control team as related to NASA's payload operations are command and control of research facilities, facilitation of command and control by remotely located operations teams, near-term timeline planning and replanning, communications to/from the ISS crew, and control of the on-board high rate data and video systems. The POIC support teams perform functions such as long range planning, crew procedure development, management and implementation of operations products, and inventory tracking of on-board experiment equipment. The size and capability of both the POIC flight control team and the support team has been a function of the workload expected and funded by the ISS Program. The POIC capability as defined by its staffing also correlates to the number of research facilities available and used on-board the ISS, as well as the amount of ISS crew time that is dedicated to research. The workload required of POIC is likewise a direct function of both these factors.

\section{A. The ISS Research Facility Capabilities}

As the ISS grew from a small outpost of only two modules starting in late 1998, to its Assembly Complete configuration in 2011, likewise the number of research facilities available for use on the ISS also increased. The first true research facilities were launched to ISS in 2001 and consisted of one International Standard Payload Rack (ISPR) dedicated to human research, and two racks configured as multi-purpose science racks that supported physical sciences, microgravity measurement, and biological research. As ISS grew during the assembly process, the ISS Program continued to add facilities and racks to increase the capability of ISS as a research platform. See Figure 2 - NASA Internal Volume Research Facilities. POIC maintains operational responsibility for these NASA research facilities, either by direct command and control, or coordinating and facilitating their operations by remotely located operations teams. In addition, NASA has installed several externally mounted research facilities for which POIC is responsible for operations. 


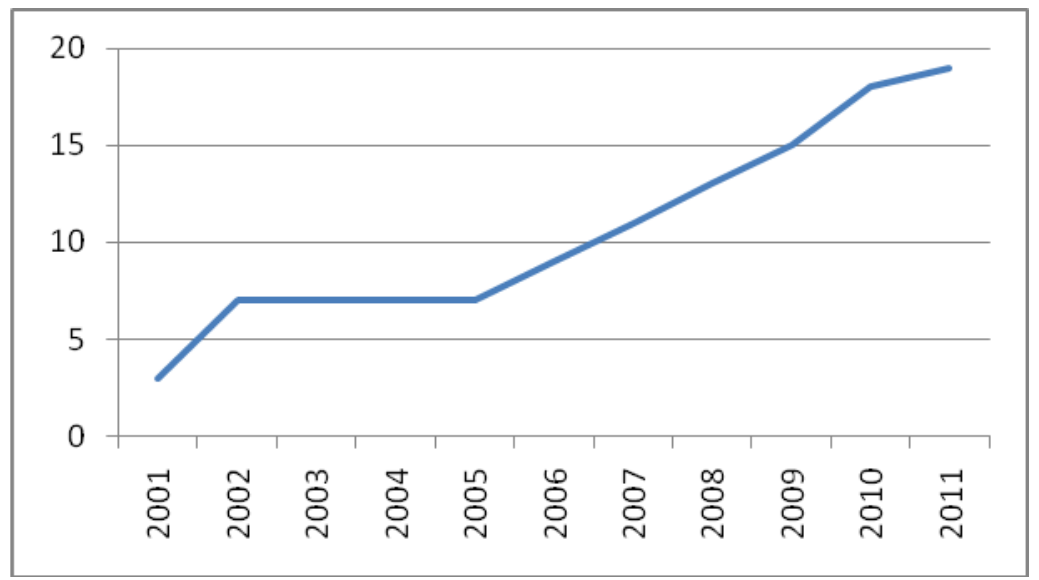

Figure 2 - NASA Internal Volume Research Facilities

\section{B. The ISS Crew Time Capability}

During the assembly phase, the on-board time available for the ISS crew to perform tasks during their workday was split among activities such as assembly, maintenance, and utilization. As assembly tasks have decreased over time, the crew time available for utilization has commensurately increased. At the start of continuous human presence on ISS in late 2000, there were three crewmembers on-board. At the official start of the ISS utilization program as implemented by POIC in early 2001; the crew size was still three people, corresponding to the nominal capability of the ISS life support systems. In early 2003, after the Space Shuttle Columbia accident, the ISS crew size was reduced to two people due to limited cargo capability to support three people on a continuous basis. ISS crew size remained at two crewmembers until mid-2006, when the Shuttle resumed flight operations and the ISS consumables such as food, clothes, and water could again be transported in necessary quantities. In mid-2009, the ISS reached sufficient size and life support capability to be able to house six crewmembers. This crew size of six people is now the nominal size for ISS and will remain so for the foreseeable future. The change in ISS crew size, from three to two to six, has had an impact on the workload required of the POIC flight control team and support team, as determined by the crew time available to conduct utilization. See Figure 3 - ISS Crew Time Dedicated to Utilization



Figure 3 - ISS Crew Time Dedicated to Utilization (hrs per week) (2011 average weekly hours as of July 2011)

The following sections of this paper provide further detail, and trace the development and evolution of POIC staffing size and content. The POIC flight control team definition was driven by operational necessity, funding 
available and estimated workload as determined by the amount of science utilization that ISS and its crew were capable of providing.

\section{Starting Out}

Historically, MSFC supported Space Shuttle Spacelab missions through the 1980's and continuing through the mid 1990's. This basis of experience proved valuable as MSFC assumed the payload operations work of the POIC, as part of the ISS Program. During the last years of the Spacelab era, MSFC's Mission Operations Laboratory formed a task team to define the newly created POIC's roles and responsibilities. The task team had many discussions about whether to begin with a new concept or to continue using the proven Spacelab model. In the end, the original POIC team definition for ISS was based upon its Spacelab predecessor, including the acronyms for the cadre positions, with some minor modifications. This early concept was documented in Revision D of the Team Definition Document for ISS Operations published in December 1999. One of the fundamental concepts at the time was NASA was responsible for the integration of the different International Partner components that comprise the ISS and MSFC was responsible for the station-wide payload operations integration across all Partners as well as NASA payload operations integration. The original concept was comprised of two complete teams: the United States Partner Control Center (US PCC) and the POIC. The US PCC was in charge of NASA science operations and the POIC was in charge of overall integrated ISS science operations, across all international partners. A couple years before operations were to start a budget cut eliminated the two team approach and the functions were combined which left POIC performing both these functions. See Figure 4 - POIC Console Positions in 2000

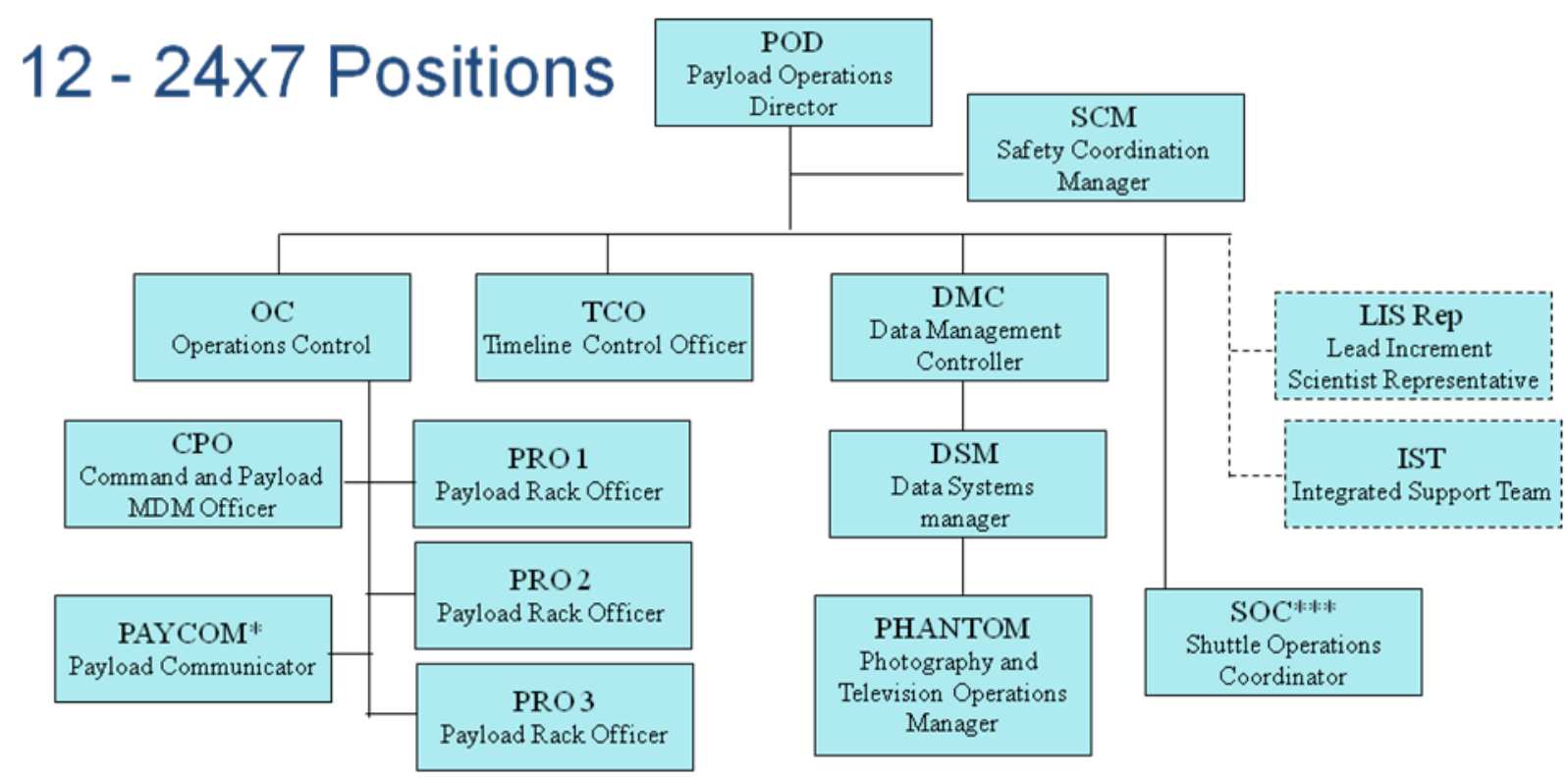

* Staffed 16x5, **** Staffed only during Shuttle Flights

Figure 4 - POIC Console Positions in 2000 (prior to starting real-time operations)

Before the POIC began real-time support in March 2001, the following changes were made to the team structure: only 1 PRO was required, and the DSM was eliminated with the DMC absorbing their duties. By August of 2001, the SCM became an on-call Safety position for NASA Payloads. See Figure 5 - POIC Console Positions in 2001 


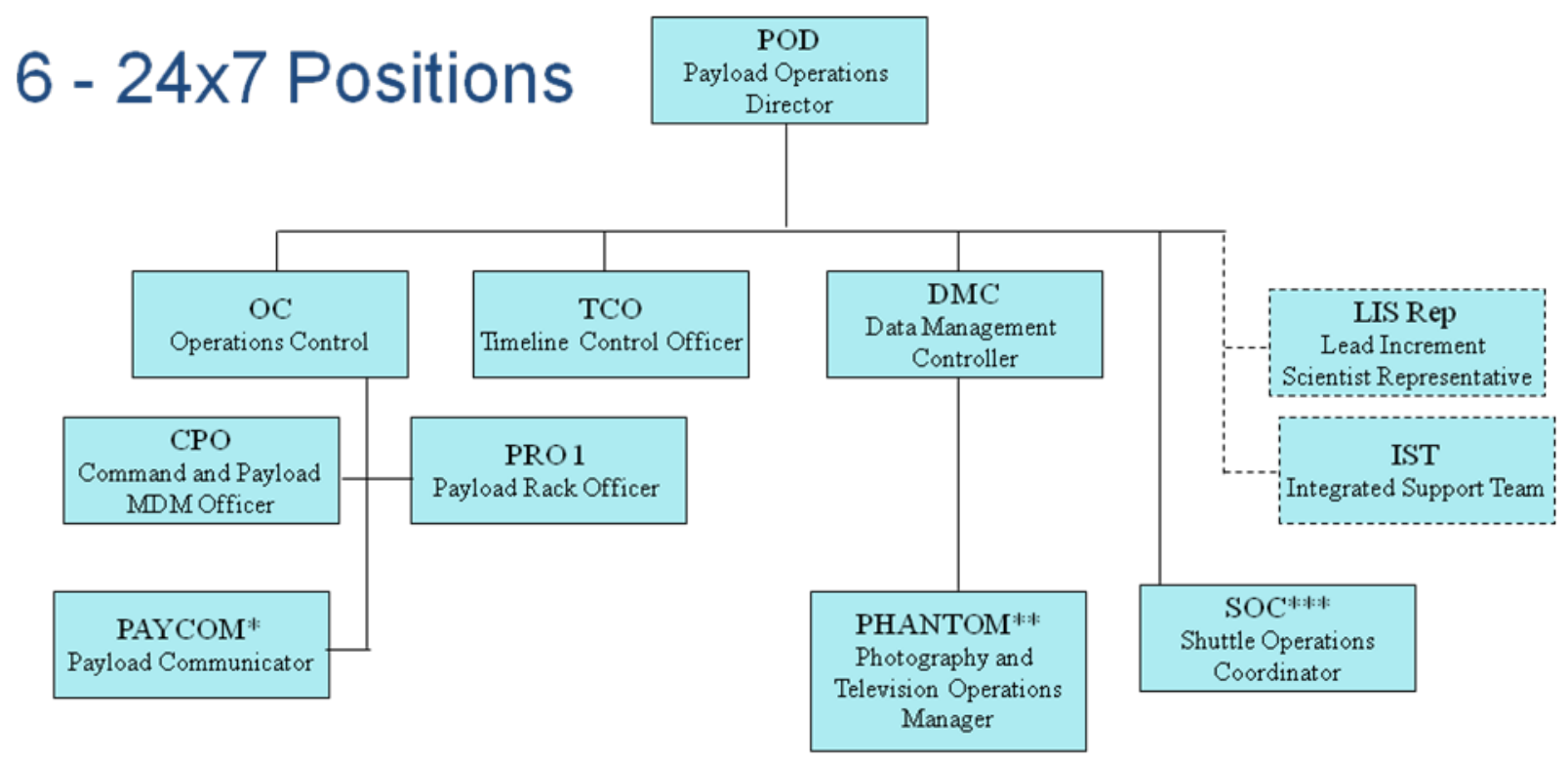

-Staffed 16x 5 , *** Staffed 16x7,

非非 Staffed only during Shuttle Flights

Figure 5 - POIC Console Positions in 2001 (at the start of real-time operations)

In mid 2001, ensuing discussions with the International Partners at each control center led to a significant reduction in the responsibility for POIC. Agreements were made for a more distributed process where each control center was responsible for its respective payloads which left POIC only responsible for NASA payload operations.

Another implemented Spacelab carry-over was the concept of defining a specific team of cadre members working together for the Increment duration. Five complete teams were defined to satisfy the $24 \mathrm{x} 7$ operations requirement. The team membership was carefully composed to ensure a balance of expertise and to avoid any known personality conflicts. Teams worked 5 days on console with 3 days off-duty before returning for their next duty period in the forward rotating cycle (days shift to swing shift to midnight shift).

During this ISS program phase, a single Increment was typically 6 months in duration. The team concept continued through Increment 4; however, we learned working together for extended periods was problematic for some team members. Eventually, the single-team concept was abandoned in 2002 during Increment 5 and each console discipline was allowed to define their specific rotational scheme.

\section{The Lean Times}

With the loss of Columbia in February of 2003, ISS operations immediately changed. With the beginning of Increment 7 there would only be two crew members on-board ISS which severely limited the amount of crew time for payload operations. Without the Shuttle flying there was also no new payload hardware arriving to operate. During this time NASA started using Russian space vehicles to transport both crew and payload hardware. The cargo limitations of these vehicles were a factor in the amount of science that could be conducted.

\section{A. Budget Cuts - Combined POIC Flight Control Positions}

In 2005, POIC was placed in a position where the scope of our four years of continuous payload operations experience on ISS could no longer be supported. POIC manpower and budget were cut approximately $38 \%$ across the board in 2005 and this took effect for fiscal year 2006. In an attempt to achieve the cuts that were directed, a new approach to the way we operated payloads on ISS was implemented. The ISS program accepted a possible 10 
to $15 \%$ inefficiency in payload operations due to the personnel cuts and lack of POIC's ability to respond quickly to recover anomalies. The technical direction received was no more than four US racks plus the Minus Eighty Degree Laboratory Freezer (MELFI) running concurrently through fiscal year 2009. Also, there would be no more than ten hours of crew time per week and there would be no support for science conducted by visiting crew members on Soyuz flights. POIC responded that there would be several noticeable changes in the way payload operations had been executed: our ability to respond to payload anomalies in real-time operations would be greatly reduced, we would not be able to react to last minute changes in crew procedures, and we would not be able to accept last minute changes to the crew's payload timeline. The 2005 budget cut, took POIC down to four 24x7 cadre positions. With this cadre reduction, we had to eliminate specialized skills and combine and merge cadre functions. See Figure 6 POIC Console Positions in 2006

The meat of these reductions were three fold; combine the planning and operations functions, combine the data and video functions, and combine the rack and commanding functions. Each of these mergers was carefully chosen, after much review and assessment, to take advantage of similar aspects of these positions which could help alleviate the additional training needed. First, the OC and TCO positions were combined into one, still called OC, but a new OC. Second, the CPO and PRO were combined into the new PRO. Third, the PHANTOM and DMC were combined into the new DMC position.

Each of these mergers was difficult and required re-training and re-certification of many console operators in a short period of time. It also involved losing good flight controllers, to get down to the reduced staffing levels, which added to the stress on all the teams at that time. Several controllers stayed up until the implementation of the changes even though they knew they were being laid off and this was a great help to the team since it freed up personnel to finish re-training. This reduction was one of the most ambitious and difficult times for the POIC but it was successfully completed without any interruption in science operations.

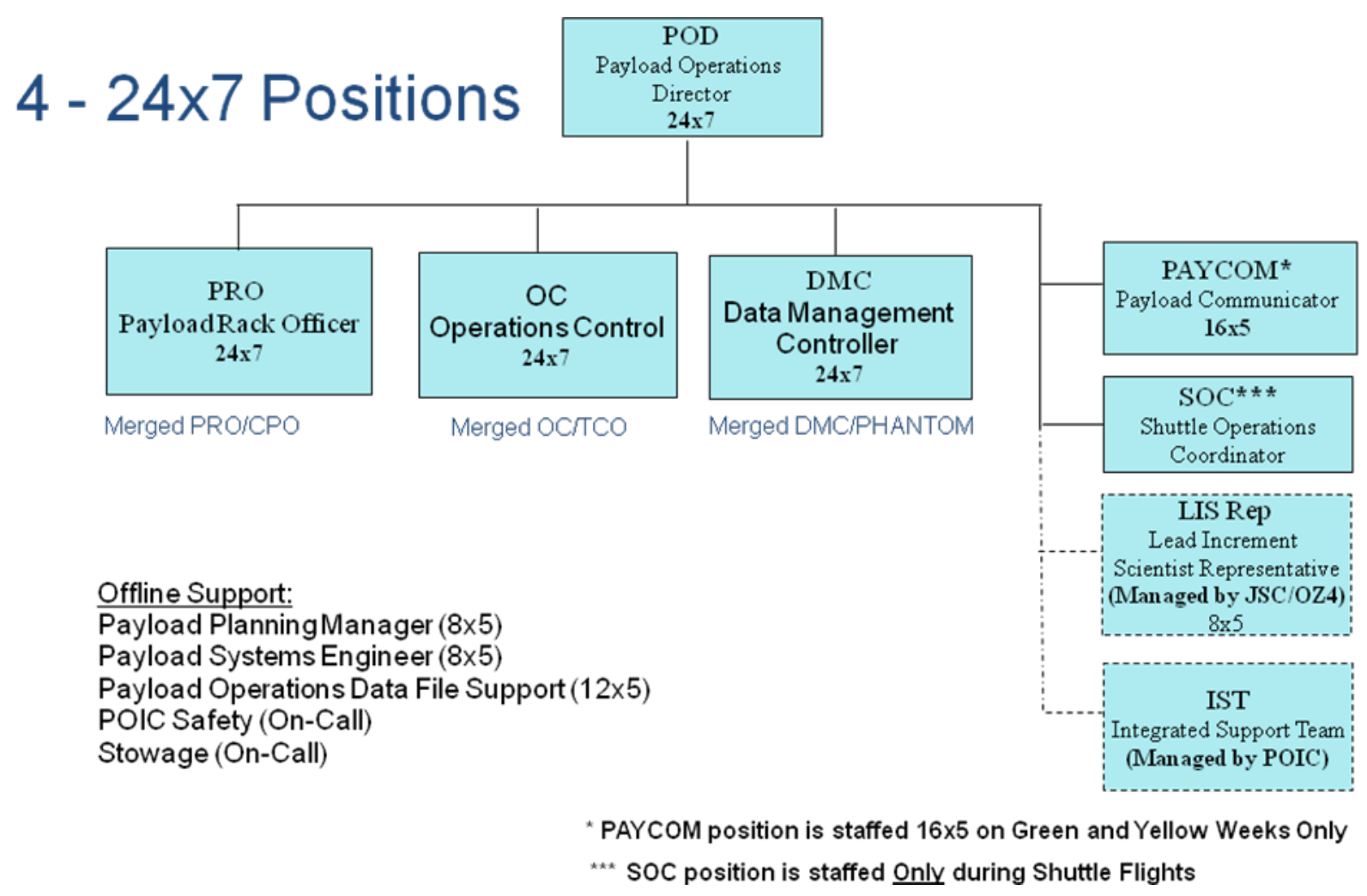

Figure 6 - POIC Console Positions in 2006 (after reductions) 


\section{Back to 3 Crew and More Room to Grow}

In 2006, the ISS occupancy increased from two back to three people. This increase in crew size along with the return of the Space Shuttle flights provided more cargo capability and crew time to perform science operations on ISS. This additional crew time and upmass resulted in a significant increase in the POIC workload. Still, however, operating on a reduced flight cadre from late 2005, efficiencies would have to be created to keep up with the increased demand for science operations.

\section{A. The A Team}

The Payload Operations Manager (POM) implemented an "A" Team concept that would be activated anytime NASA scientific operations exceeded $20 \mathrm{crew}$ hours in one week. The A Team consisted of one person from each flight control discipline who was on-call during the standard work week for supporting the POIC team. The A Team concept provided the additional support POIC needed to cope with the work load, while still remaining flexible to not be over-staffed during weeks when there were fewer hours available for science operations. This team supplemented the flight room cadre by performing daily paperwork tasks such as timeline reviews, operational change requests, and day to day contact with the payload developers.

\section{B. Addition of International Partner Modules}

During 2008, the ESA Columbus and JAXA Kibo modules were installed on the International Space Station. See Figure 7 - ISS Partner Modules. ISS occupancy also ramped up to the full complement of 6 people. With these additions, came a new capability for expanded payload opportunities to be executed by the POIC. Human Research Facility (HRF) Racks \#1 and \#2 as well as EXPRESS Rack 3 were transferred to the Columbus module, and the MELFI 1 and EXPRESS Racks 4 and 5 were transferred to the Kibo. With NASA payloads distributed in International Partner modules, the number of operational and systems interfaces increased. More crew onboard meant even more time for science and an increased requirement to command and control NASA payload racks. A full-time Assistant Payload Operations Manager (APOM) was created to assist with the planning and preparation for the additional crew time available for payload operations.

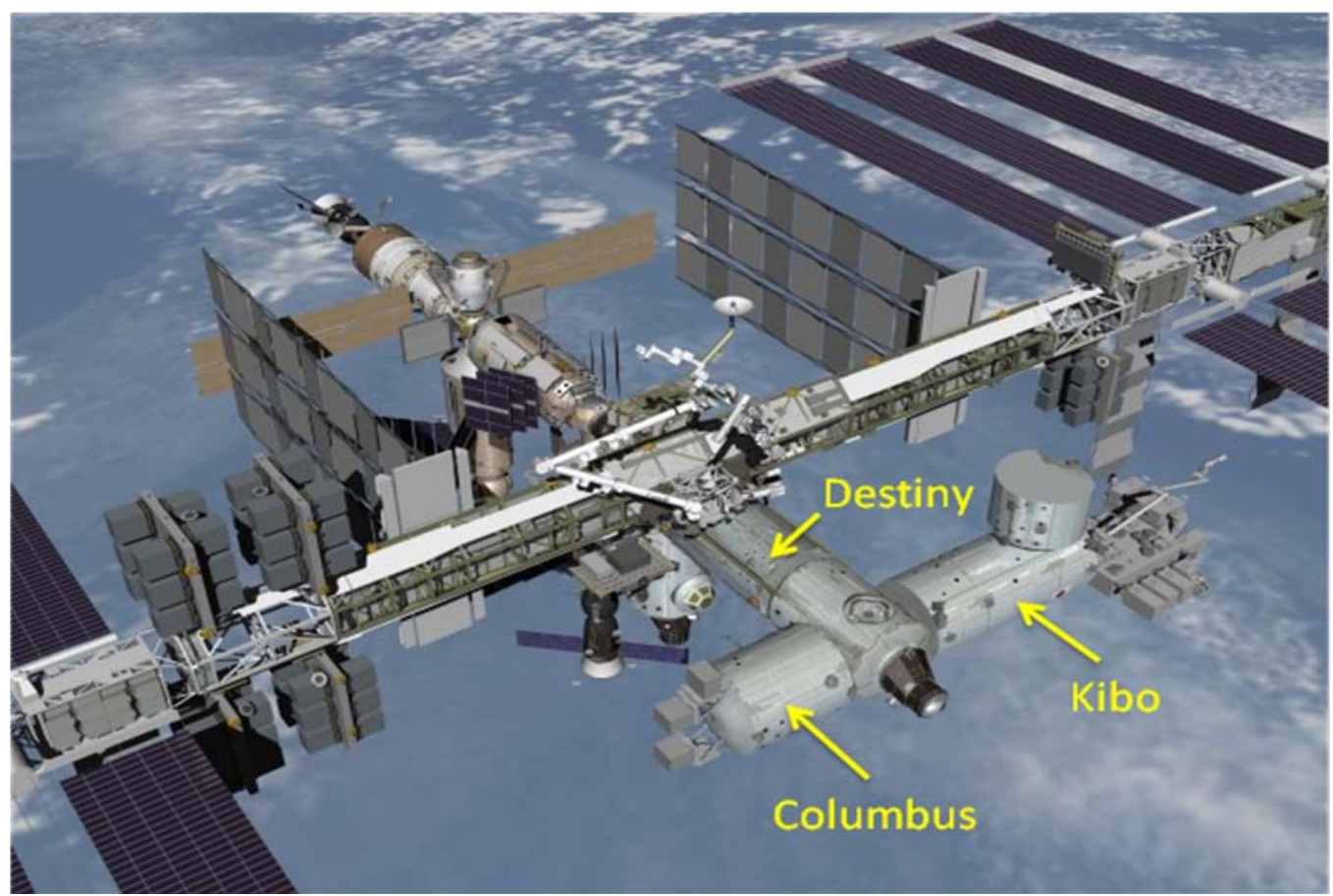

Figure 7 - ISS Partner Modules 


\section{More Payloads and More Help}

In early 2008, it was realized that payload operations were going to change with the increase from 3 to 6 crew onboard ISS. Increasing the crew size meant more opportunities each week to perform payload operations but also meant more possibilities for concurrent payload operations and a heavier team workload. It was also becoming apparent that POIC needed to be able to operate more payload facilities (racks) simultaneously because the current limit was affecting how much science was being accomplished (only 5 payload racks powered at the same time). With the POIC team structure at that time, more real-time operations would put more stress on the team and jeopardize some of the routine/preparation type tasks that need to be done every day. To help the team focus on science operations, an expanded team structure was proposed in 2008 and implemented in mid 2009. See Figure 8 POIC Console Positions in 2009

This team expansion included three basic changes: taking some change processing type tasks off of the POD, adding additional support for timeline review/changes, and adding additional rack officer support. First, a new position was created to shepherd change requests thru the system, the Change Traffic Engineer (CTE). Already certified/seasoned OCs were used for the new CTE position since they were very familiar with the change request process and to help cut down on training needed prior to implementation. Second, a support position was created to help the OC with review and re-planning type tasks, OC Support (OCS), and this position was also staffed with certified OCs to cut down on training needed. This gave the OC the flexibility to assign almost any OC task to the OCS since they were already trained on all aspects of the OC job. This allowed the OC to focus on real-time operations while the OCS was working on reviewing the next day's plan, reviewing change requests, or submitting timeline changes for subsequent days. Third, PRO staffing was increased so that two PROs were on console Monday thru Friday (two shifts per day) to support increased payload rack operations. Even with this increased PRO support, there were still limits on how many concurrent racks could operate and when rack activations or deactivations could occur (seven racks powered continuously and no rack activations or deactivations when only one PRO on console).

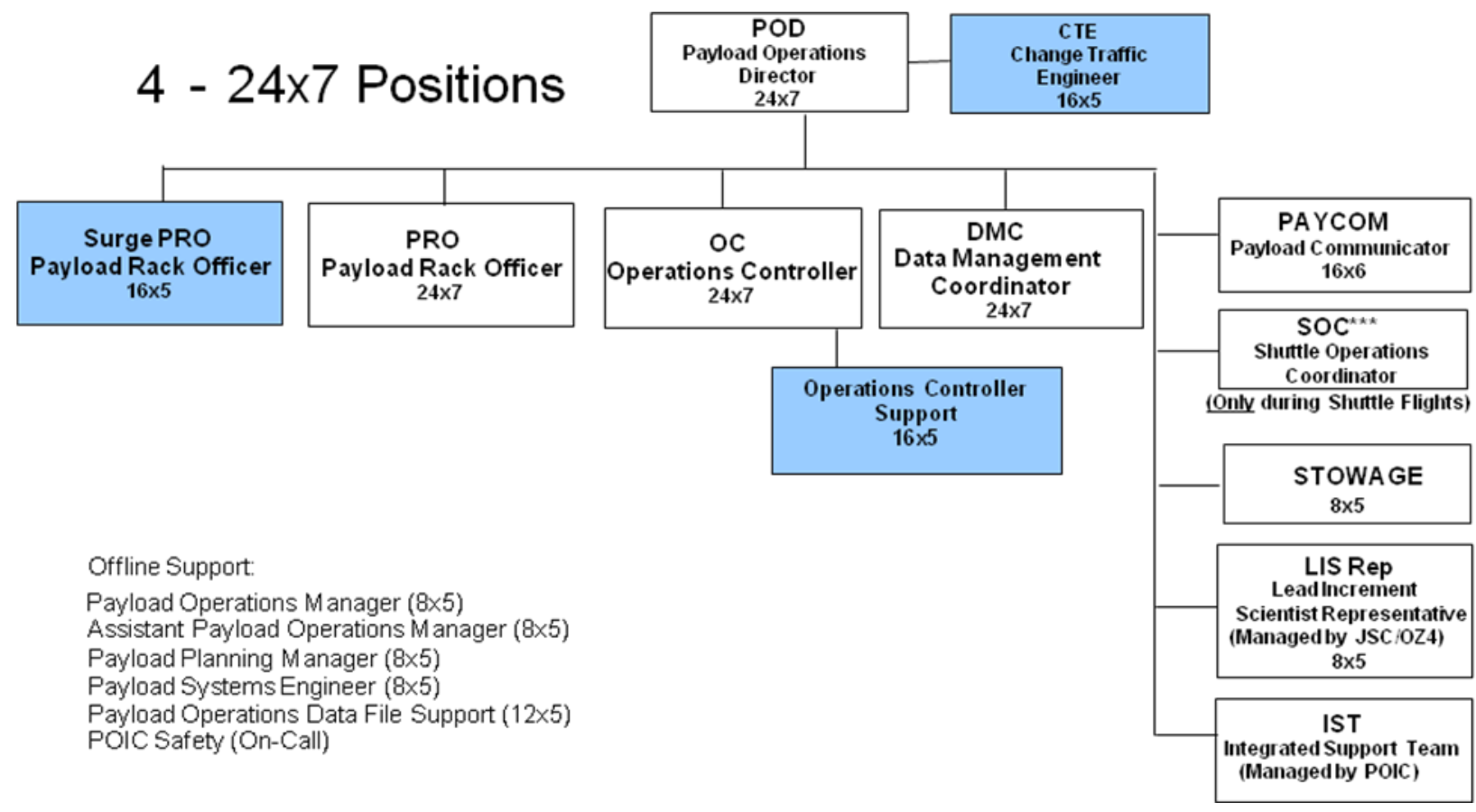

Figure 8 - POIC Console Positions in 2009 (after additions)

It was quickly apparent that these changes helped alleviate the team's workload. Taking the majority of change traffic off of the POD was a real benefit since it allowed the POD more time to focus on real-time operations and not 
have to shift gears to focus on an upcoming activity change 2 weeks in the future. It also put the approval of changes on the management team in the back rooms who many times have a better grasp on the upcoming big picture of science operations. The OC having support for their duties was an easy split since many of their tasks include reviewing and submitting changes for operations within the next week and these types of tasks could easily be done by someone other than the OC that was focused on real-time operations. Having the extra PRO support definitely allowed the POIC to perform more concurrent rack operations which lead to more science getting accomplished.

\section{A. Our Time Had Come}

In late 2009 discussions began which focused on ISS assembly complete fast approaching and science finally getting more crew time. The ISS Program stated that it wanted at least 35 to 40 hours of crew time each week going to payload operations. Work began to develop concepts for increasing the amount of science operations that POIC could handle simultaneously to support this increase in crew time. This work also included removing POIC staffing as a constraint to simultaneous facility operations which would let us run as many science racks as the ISS resources would allow. In mid 2010 concepts were presented and additional staffing was granted to increase POIC's science throughput. In the fall of 2010 three tiger teams were created to flush out all the details of this new cadre structure with the goal of implementing these changes in September 2011.

This expansion was broader in scope than the previous one but in many ways took those previous concepts to their inevitable final state. The earlier addition of CTE to handle change traffic was taken even further by creating an entire new team that had change traffic as one of its core responsibilities. This new PREP Team's (Product Readiness and Enhancement for Payloads) main function was to get all the products needed for science operations ready to go. The team was made up of the leads in each discipline which allowed for proper review of products and change requests. The other main objective of this new team was to take the daily timeline review task off of the team that was executing science which allows the execution team more time to focus on operations, giving them the ability to operate more science each day. The discipline leads had already started having a weekly timeline review a year or so earlier to help improve timeline quality so creating a new team which would do this task every day was a logical progression of that earlier idea. See Figure 9 - POIC PREP Room

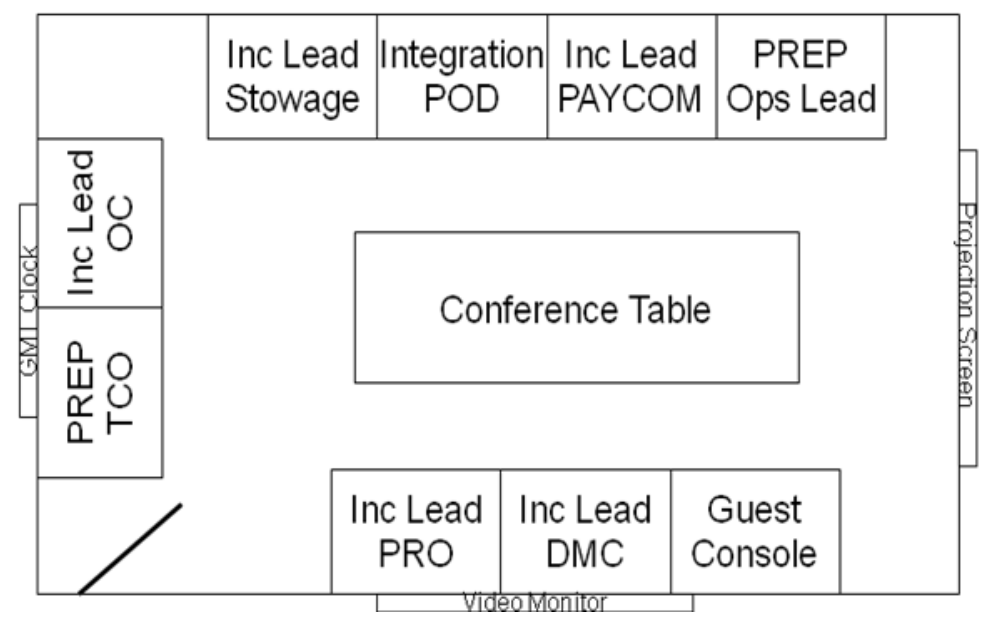

Figure 9 - POIC PREP Room

The other major change to POIC staffing in this expansion was the total splitting apart of the OC tasks - back to what they had originally been years earlier with the TCO. When the OC and TCO tasks were combined back in 2006 it was quickly evident that the combination of those tasks was ambitious and not really optimal. The creation of OCS was an interim approach to alleviating the OC workload but adding the full TCO position back to the POIC cadre will allow the OC to focus on science operations while having a dedicated position working all the planning changes/issues. This OC/TCO compliment really unleashes some operations capability for POIC to perform more 
science each day and was a natural evolution for the POIC cadre. See Figure 10 - POIC Console Positions in late 2011

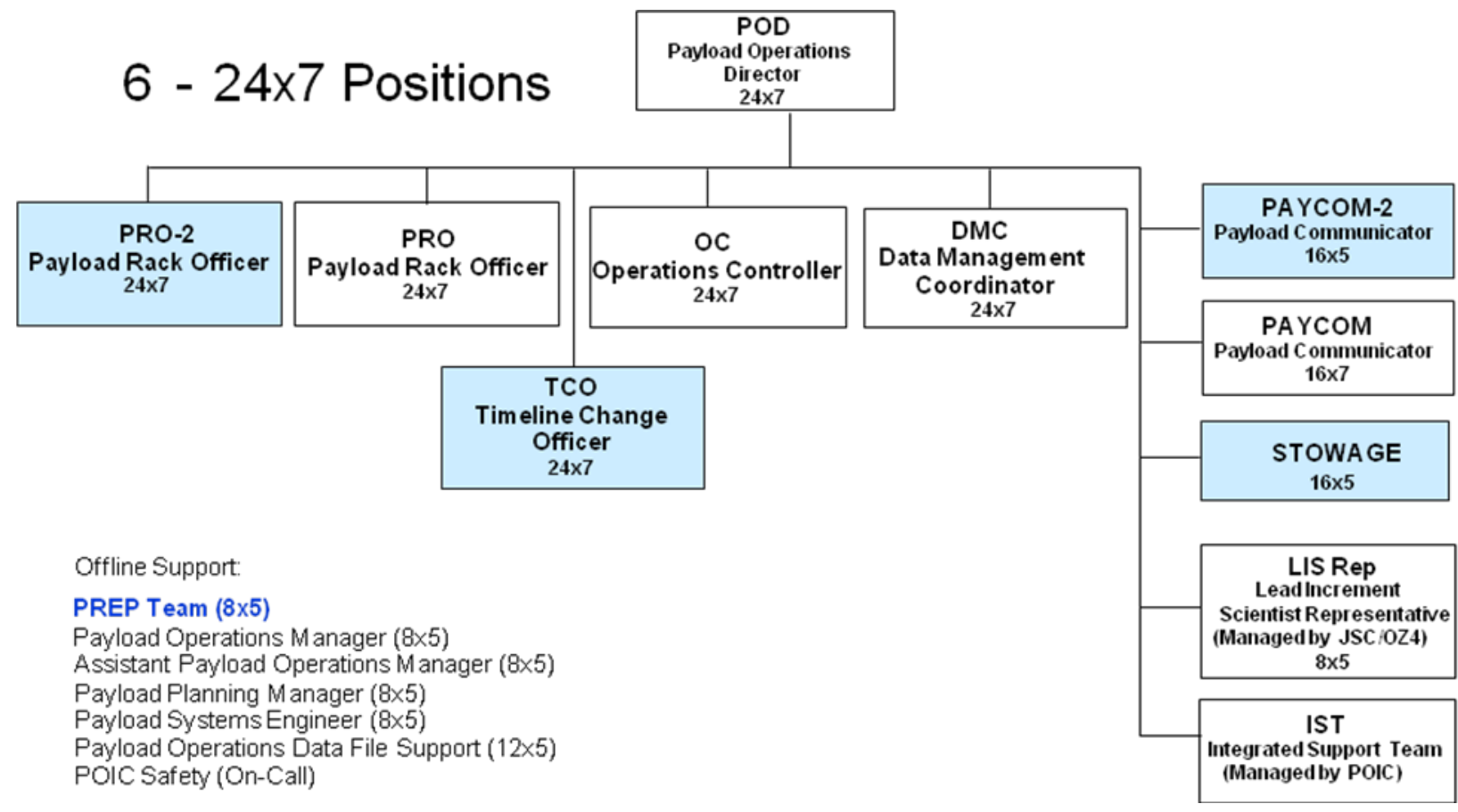

Figure 10 - POIC Console Positions in late 2011 (after additions/deletions)

A few more staffing increases were made during this time: PRO staffing was increased to two full time PROs on each shift, POIC Stowage staffing was increased to two shifts per day during the work week, and PAYCOM staffing was increased to allow for two PAYCOMs while the crew is awake. The increase in PRO support alleviated any constraints POIC was putting on how many facilities could be operated simultaneously. Extra Stowage support was needed while the crew was working on science since the amount of stowage on ISS has grown immensely and being able to find what you need when you need it certainly makes more efficient use of the crew's time. Having a second PAYCOM available during science operations can greatly increase the focus and response to crew operations and questions. This capability can eventually allow the POIC to discuss multiple science operations at the same time, which is very important when two or three crew can be working on science at the same time. Dual discussion with the crew can be a tricky thing when the rest of the POIC is trying to keep up with two conversations. So obviously this concept will need testing and practice before it is perfected but simultaneous payload operations is where the POIC needs to be and we will get there.

At the time of this writing, the new PREP Team and additional staffing in POIC concepts are complete and only a few documentation details remain. POIC is ready and will begin these new concepts in mid September 2011 with the start of Increment 29. Only time will tell how effective these changes will be but all signs point to a very successful and important change for the POIC that will truly let us all utilize ISS as it was meant - as a science laboratory.

\section{Conclusion}

The capabilities and limitations of the POIC to conduct payload operations on-board the International Space Station have fluctuated significantly during the first decade of support. Some of these changes can be attributed to the initiation of performing scientific activities before Assembly Complete was achieved. During this period, payload operations were assigned a much lower priority and the initial staffing concept was reduced to correspond. 
Science activity was also limited during extended periods when the Space Shuttle launches were suspended. The fluctuating annual budget for NASA also had an impact on the resources and manpower available for POIC support. With each change came a new learning experience or incentive that allowed us to streamline and improve processes and products for greater efficiency.

With the recent retirement of the Space Shuttle fleet, the last major components have been installed and the ISS is deemed to be completed. The new focus by the ISS Program is to fully utilize this orbiting science platform to perform at least 35 hours of science every week. Within the next year, we expect upgrades to the payload data and video system hardware to be completed that will double the current downlink bandwidth. In this same period of time, the on-orbit software will be upgraded to increase the number of concurrently active payload racks and facilities. There is no doubt that the future will bring further changes due to improved technology or other unknown drivers that will affect the POIC's capabilities and limitations to conduct payload operations. The POIC is poised to accept these challenges and to continue our tradition of successful completion of science on ISS. 